\title{
Assessing the Quality and Reliability of Health Information on ERCP Using the DISCERN Instrument
}

\author{
Kaicker $\mathrm{J}^{1}$, Dang $\mathbf{W}^{2}$ and Mondal $\mathrm{T}^{3 *}$
}

${ }^{1}$ Michael G Degroote School of Medicine, McMaster University Medical Center, 1280 Main Street West, Hamilton, Ontario, L8S 4K1, Canada

${ }^{2}$ University of Ottawa School of Medicine, 451, Smyth Rd., Ottawa (Ontario) Canada K1H 8M5

${ }^{3}$ Department of Pediatric Cardiology, McMaster University Medical Center, 1280 Main Street West, Hamilton, Ontario, L8S 4K1, Canada

\begin{abstract}
Introduction: Endoscopic retrograde cholangiopancreatography (ERCP) is a technique that uses both fluoroscopic imaging and luminal endoscopy to help diagnose and treated pancreatobiliary pathologies. With increasing use of the internet to gain medical competence, the purpose of this investigation is to assess the quality of online health information about the ERCP procedure.

Methods: ERCP related websites were assessed using the Google, Yahoo and Bing search engines. The DISCERN instrument and JAMA benchmarks were used to critically assess each websites. A total of 60 websites were reviewed with 7 websites excluded based on the exclusion criteria established. After removal of duplicate websites, a total of 24 unique websites were assessed. Websites that were exclusively visually based, could be modified by the general population and banner advertisements were not included in the investigation.

Results: The average DISCERN score was 42.2 (9.1) for the 24 unique websites assessed. Using the JAMA benchmarks, appropriate authorship and attribution of references were seen in $25 \%$ and $29 \%$ of websites respectively. Currency, which ensures that website developers provide dates when content is posted was only seen in $13 \%$ of cases with disclosure, indicating potential conflict of interest, seen in $17 \%$ of cases.

Conclusion: The overall quality of websites discussing ERCP is of low to moderate quality. The highest scoring websites were concise, with clear aims and described the procedures with benefits and associated risks. The lowest scoring websites failed to adequately reference information and describe additional treatment options. Websites that appeared first on each search engine for ERCP did not necessarily score better, demonstrating the importance of providing patients with high quality resources. Future investigation should use additional critical appraisal tools to assess ERCP related websites and assess the impact of varied formats (text, animations) on the quality of information provided.
\end{abstract}

Keywords: ERCP; Endoscopic retrograde cholangiopancreatography; DISCERN Instrument; JAMA Benchmark

\section{Introduction}

Canadians have been increasing using the internet over the past decade with recent figures estimating as many as 25 million users [1]. Within these users are many that use the internet to seek health related information. This use has been found to be inversely associated with age, meaning that web based health information will be ever more important in the future [2,3]. Recent investigations have found that patients attending gastroenterology clinics report internet use for health conditions between 42 and $92.6 \%$ [1]. The information sought is generally about the etiology of conditions and treatment options.

An investigation conducted by Google has demonstrated that $86 \%$ of physicians use the internet to collect medical or treatment information [4]. While many physicians conducted future research (48\%) after the initial search, about a third admitted to changing a potential treatment plan after consulting the internet [4]. Inaccurate, non evidence based and biased information can potentially compromise patient care. It is therefore important to assess the quality of online information available to physicians and patients.

\section{Endoscopic retrograde cholangiopancreatography}

Endoscopic retrograde cholangiopancreatography (ERCP) is a technique that uses both fluoroscopic imaging and luminal endoscopy to diagnose and treated pancreatobiliary pathologies [5]. Indications for this procedure include: Assessment and treatment of biliary obstruction secondary to choledocholithiasis, treatment of choledocholithiasis identified during cholecystectomy and assessment and treatment of bile duct strictures [5]. The long name of this procedure in combination with unfamiliarity with the pancreatobiliary system can results in many patients and health professionals consulting the internet.

\section{Quality assessment instruments}

Two solutions to address the issues of accuracy and reliability of online health information have been designed, DISCERN instrument and JAMA benchmarks. The DISCERN questionnaire is a valid and reliable instrument for analyzing written consumer health information [6]. It is comprised of 16 questions assessing both the reliability of the resource and quality of information about the treatment choice. The JAMA benchmarks provide a means for qualitatively assessing websites for the presence of authorship, attribution, currency and disclosure [7]. Thus, the purpose of this investigation is to assess the quality of online health information about the ERCP procedure.

*Corresponding author: Dr. Tapas Mondal, Associate Professor, Department of Pediatric Cardiology, McMaster University Medical Center, 1280 Main Street West, Hamilton, Ontario, Canada, L8S 4K1, Tel: (905) 521-2100 ext 75242; Fax: (905) 521-7914; E-mail: mondalt@mcmaster.ca

Received August 11, 2013; Accepted October 11, 2013; Published October 14, 2013

Citation: Kaicker J, Dang W, Mondal T (2013) Assessing the Quality and Reliability of Health Information on ERCP Using the DISCERN Instrument. Health Care Current Reviews 1: 104. doi: 10.4172/2375-4273.1000104

Copyright: @ 2013 Kaicker J, et al. This is an open-access article distributed unde the terms of the Creative Commons Attribution License, which permits unrestricted use, distribution, and reproduction in any medium, provided the original author and source are credited. 


\section{Methods}

This investigation used the DISCERN instrument and JAMA benchmark to critically appraise the online health information pertaining to ERCP. The three most popular search engines from the Nielsen/Net Ratings were used for this investigation including Yahoo, Google, and Bing [8,9]. The search engines (www.google.com, www. yahoo.com, and www.bing.com) were assessed on July 29, 2013, in English. The first 20 hits from each search engine were examined by using the search term "ERCP" for a total of 60 websites. The limit for assessing hits was based on previous investigations that have shown that individuals typically examine the first page of a search engine, which typically contains ten hits [10].

\section{Inclusion/Exclusion criteria}

Websites were included in the investigation if they provided information that related to the ERCP procedure. Furthermore, websites that were exclusively visually based, completed scientific articles at advanced level for many patients or did not provided information about the ERCP procedure were excluded. Websites that could be modified by the general population were also not considered in this investigation. Moreover, banner advertisements or sponsored links were not included. Duplicate sites across and within search engines were also removed from the analysis. A total of 60 websites were reviewed with 7 websites excluded based on the exclusion criteria established. After removal of duplicate websites, a total of 24 unique websites were assessed. This methodology is summarized in Figure 1.

\section{DISCERN instrument}

The DISCERN instrument is a reliable tool for assessing written consumer health information. It is the first standardized quality index of consumer health information that can be used as a critical appraisal tool to evaluate health information by not only health professionals, but also by patients and the general population. It was developed with the input of an expert panel, health information providers and patients from a self-help group.

The DISCERN questionnaire is comprised of 16 questions on a rating scale of 1 to 5 . A score of 1 states a definite NO and 5 a definite YES [6]. Any rating between 2 to 4 suggests that some elements of the question are present. Section 1 (Questions 1 to 8 ) assesses reliability whereas Section 2 (Question 9 to 15 ) focus on the quality of information

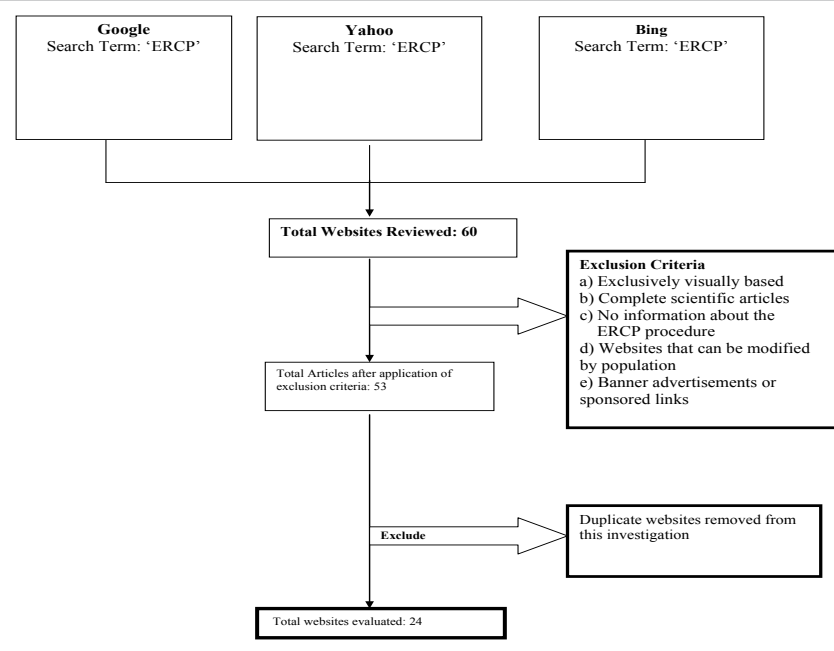

Figure 1: Consort Diagram for Website Selection. about treatment choices. Section 3 (question 16) provides an overall quality rating independent of the previous 15 questions [6].

\section{JAMA benchmarks}

The JAMA benchmarks are a series of four criteria established by the Journal of the American Medical Association. Authorship refers to the website appropriately stating the author's name, affiliations and credentials. Attribution refers to effective reference of content presented throughout a website. Currency ensures that website developers provide dates when the content is posted and then updated. Finally, the disclosure category demonstrates site 'owner' are highlighted with any potential conflict of interest stated.

\section{Data abstraction}

All the search terms listed above were inputted into the search engines chosen for this investigation. We independently assessed the quality of each website using the DISCERN instrument. Assessment was not limited to the initial page, but the entire website. A score out of 5 was generated for each of the 16 questions. Individual question scores were used to generate a score from 80 . The mean score, standard deviation (SD) between the two reviewers was presented. Descriptive analyses were carried out using SPSS software Version 17 (Chicago, IL, USA).

\section{Results}

The 24 unique websites assessed in this investigation were ranked according to their total DISCERN scores. Each website was independently assessed by each reviewer with the mean score between the two reviewers presented. The scores from a maximum of 80 ranged from 25 to 65 . The average DISCERN score was 42.2 (9.1). The five highest scoring websites found are summarized in Table 1.

Questions where websites consistently did well included providing appropriate aims of the website, relevant information pertaining to ERCP and describing the risks of the treatment. On the contrary, websites were consistently deficient in providing evidence of clear source of information used and providing evidence of the date of when the information used was produced. The websites also failed to consistently describe the effect for patients in which treatment was completed and whether or not additional treatment options were present. Individual DISCERN question scores based on the websites assessed can be seen in Figure 2. Finally, when assessing the websites using the JAMA benchmarks, the majority of websites lacked authorship, attribution of reference and disclosure (Table 2).

\section{Discussion}

During ERCP, an endoscope is passed through the mouth to the duodenum with the Ampulla of Vater identified [5]. Radiographic

\begin{tabular}{|c|c|c|}
\hline Name & Address & DISCERN Score \\
\hline $\begin{array}{c}\text { John Hopkins } \\
\text { Medicine }\end{array}$ & http://pathology.jhu.edu/pc/ercp.php & 65 \\
\hline $\begin{array}{c}\text { Guy's and St } \\
\text { Thoma's NHS }\end{array}$ & $\begin{array}{r}\text { http://www.guysandstthomas.nhs.uk/resources/ } \\
\text { patient-information/acute/gi-surgery/having-an- } \\
\text { ercp.pdf }\end{array}$ & 61.5 \\
\hline Healthlink BC & $\begin{array}{r}\text { http://www.healthlinkbc.ca/kb/content/ } \\
\text { medicaltest/tu3442.html }\end{array}$ & 55.5 \\
\hline Patient.co.uk & http://www.patient.co.uk/health/ercp & 51 \\
\hline Upto Date & $\begin{array}{r}\text { http://www.uptodate.com/contents/ } \\
\text { ercp-endoscopic-retrograde- }\end{array}$ & 52 \\
\hline
\end{tabular}

Table 1: Top Five Websites. 


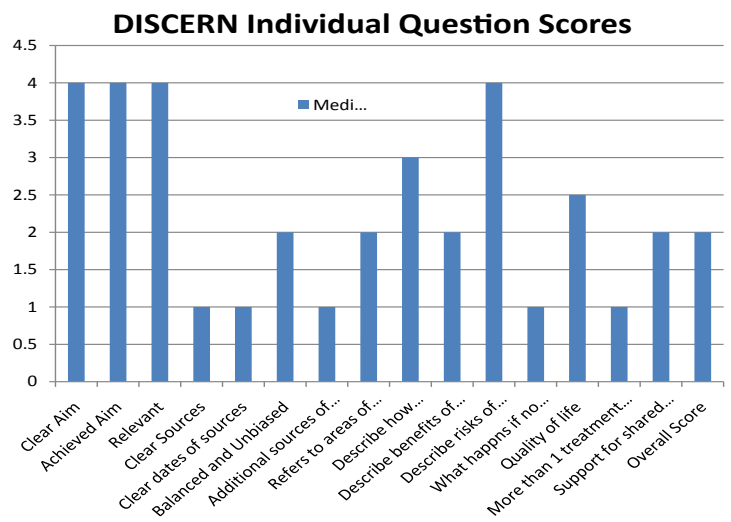

Figure 2: DISCERN instrument individual scores.

\begin{tabular}{|c|c|c|}
\hline JAMA Benchmarks & Number of websites(n=24) & Percentage (\%) \\
\hline Authorship & 6 & 25 \\
\hline Attribution of References & 7 & 29 \\
\hline Currency & 13 & 54 \\
\hline Disclosure & 4 & 17 \\
\hline
\end{tabular}

Table 2: Websites meeting JAMA Benchmarks.

contrast material is injected into the bile duct and pancreatic duct using fluroscopy. The sphincter of Oddi can subsequently be opened using the technique of endoscopic sphincterotomy [5]. Stones can be retrieved from the ducts, biopsies performed, strictures can be dilated or stented [5]. This complex procedure can be daunting to many patients, who can utilize the internet to learn more about the procedure. Typically, the three most common search engines, Google, Yahoo and Bing are utilized. It is therefore imperative to assess the quality of online ERCP related information. This study found an average DISCERN score of 42.2 (9.1) demonstrating moderate quality websites with some significant shortcomings.

The online ERCP related health information consistently scored well in the categories of providing appropriate aims of the website, relevant information pertaining to ERCP and describing the risks of the treatment. By providing specific aims of the information and relevant information, patients and health providers are more likely to read the entire content of the website and recommend it to colleagues and friends. In addition, since many websites assessed were affiliated with either a university or hospital, it is imperative to provide patients with information about potential risks of treatment. This allows for informed patient consent and can ease anxious patients, unfamiliar with the procedure.

It is importance to understand the deficiencies found consistently on ERCP websites using both the DISCERN and JAMA benchmarks. Many websites failed to demonstrate appropriate citation of information and dates when information was updated. This finding is consistent with previous studies which have found that the online information can offer unbalanced view with little or poor referencing to scientific data [11]. Citations are imperative to provide credit to the individual who originated the information. Similarly, providing information about the author and any potential conflict of interest with regards to the information presented provides accountability. Information from authors who are health professionals can be considered more credible due to their expertise in the field.

Furthermore, websites also failed to consistently describe any additional treatment options or the prognosis for patients if the ERCP was forgone. In the era of patient centered health care, appropriate informed consent for procedures is imperative. In understanding patient consent, capacity refers to the ability to understand information relevant to a treatment decision and appreciate foreseeable consequences of a decision or lack of decision. Two important components of this include the ability to understand the alternatives (if any) to the proposed treatment and to understand the option of refusing treatment and its consequences [12]. While the internet has the capability to improve informed consent, allowing patients to become more aware of procedures, many websites are failing to help accomplish this.

Furthermore, this investigation demonstrated that no relationship between rank order of hits generated by the search engines and quality scores. Websites of higher quality, as determined using the DISCERN instrument, did not necessarily appear as higher hits in any of the search engine used. This is not consistent with findings seen in a similar study, which showed a clear correlation of Internet site quality with search ranking [13]. It is imperative for health professionals to therefore provide patients with the best available resources for a given topic.

\section{Strengths and Limitations}

This was the first investigation to assess online health related information about Endoscopic Retrograde Cholangiopancreatography (ERCP) using both the DISCERN instrument and JAMA benchmarks. The top websites found can be recommended to patients by health professionals. Previous investigations have also used the DISCERN instrument to assess anxiety disorders, post herpetic neuralgia and gastrointestinal pathologies such as Inflammatory Bowel Disease $[1,14,15]$. This investigation selected a procedure that is unfamiliar to many patients and physicians. Unfamiliar procedures and biologic systems are more likely to be searched online to gain general competency.

There are also some limitations to this investigation. The internet and search engines are dynamic processes that constantly change. The websites assessed in this investigation may not necessarily reflect the information available to patients at another point in time. Secondly, only the top 20 website sites were selected from each search engine. While previous studies have suggested that individuals typically utilize the first page of search engine, high quality websites may not have been assessed. Furthermore, only North American and websites in English were considered.

\section{Conclusion}

The overall quality of websites discussing ERCP is of low to moderate quality. The highest scoring websites were concise, with clear aims and described the procedures with benefits of risks highlighted. The lowest scoring websites failed to adequately reference information and describe additional treatment options or the prognosis for patients if the ERCP was not done. Websites that appeared first on each search engine for ERCP did not necessarily score better than latter sites, demonstrating the importance of providing patients with high quality resources. Due to the broad nature of the instrument and extensive questions, it can be used to assess many other health conditions. Comparisons can be made to determine the quality of this information and were potential deficiencies lie, in contrast to this investigation. A greater awareness of this instrument for the general public is importance so that individuals can start to critically assess the websites they are using for medical information. Future investigation should use 
Citation: Kaicker J, Dang W, Mondal T (2013) Assessing the Quality and Reliability of Health Information on ERCP Using the DISCERN Instrument. Health Care Current Reviews 1: 104. doi: 10.4172/2375-4273.1000104

additional critical appraisal tools to assess ERCP related websites and assess the impact of varied formats (text, animations) on the quality of information provided.

\section{References}

1. Tangri V, Chande N (2010) Quality of Internet based information on gastrointestinal diseases. Can J Gastroenterol 25: 93-96.

2. Cima RR, Anderson KJ, Larson DW, Dozois EJ, Hassan I et al. (2007) Internet use by patients in an inflammatory bowel disease specialty clinic. Inflamm Bowel Dis 13: 1266-1270.

3. Van Der Marel S, Duijvestein M, Hardwick JC, Van Den Brink GR., Veenendaal R, et al. (2009) Quality of Web based information on Inflammatory Bowel Disease. Inflamm Bowel Dis 15: 1891-1896.

4. Connecting with physicians online: Searching for answers.

5. Longo DL, Fauci AS, Kasper DL, Hauser SL, Jameson JL, et al. (2011) Harrison's Principles of Internal Medicine. (18thedn), McGraw-Hill Companies: New York.

6. Charnock D, Shepperd S, Needham G, Gann R (1999) DISCERN: an instrument for judging the quality of written consumer health information on treatment choices. J Epidemiol Commun Health 53: 105-111.
7. Silberg WM, Lundberg GD, Musacchio RA (1997) Assessing, controlling, and assuring the quality of medical information on the Internet: Caveat lector et viewer-let the reader and viewer beware. J Am Med Assoc 277: 1244-1245.

8. Nielsen/NetRatings (2010) Netview usage metrics.

9. Bar-llan J (2005) Comparing rankings of search results on the web. Inform Process Manag 41: 1511-1519.

10. Elwyn G, Edwards A, Kinnersley $P$ (1999) Shared decision-making in primary care: the neglected second half of the consultation. Br J Gen Pract 49: 477-482.

11. Cline RJW, Haynes KM (2001) Consumer health information seeking on the Internet: the state of the art. Health Educ Res 16: 671-692.

12. Etchells E., Sharpe G, Elliott C, Singer PA (1996) Bioethics for CliniciansCapacity. CMAJ 155: 657-661.

13. Than BHJ, Kostapanagiotou K, Jilaihawi AN (2009) A review of mesothelioma information on the World Wide Web. Thorac Oncol 91: 102-104.

14. Ipser JC, Dewing S, Stein DJ (2007) A systematic review of the quality of information on the treatment of anxiety disorders on the Internet. Curr Psychiatry Rep 9: 303-309

15. Hallingbye T, Serafini M (2011) Assessment of the quality of postherpetic neuralgia treatment information on the Internet. J Pain 12: 1149-1154. 